\title{
Numerical Simulation of the Flow Field in the Mixing
}

\section{Section of a Screw Extruder by the Lattice Boltzmann}

\section{Model}

\author{
J.M. Buick ${ }^{1}$, J.A. Cosgrove ${ }^{2}$ \\ ${ }^{1}$ Physics and Electronics, School of Biological, Biomedical \\ and Molecular Sciences, University of New England, NSW 2351, Australia \\ ${ }^{2}$ School of Physics, The University of Edinburgh, EH9 3JZ, Scotland, UK
}

November 10, 2005

keywords: Numerical Simulation; Lattice Boltzmann model; Screw Extruder; Fluid Mixing; Flow Field; Velocity Profile

Manuscript Reference: AB/2004/001256

\begin{abstract}
The single-screw extruder is commonly used in polymer processing where the performance of the mixing section is significant in determining the quality of the final product. It is therefore of great interest to simulate the flow field in a single-screw extruder. In this paper the simulation is performed using the lattice Botlzmann model giving a fast
\end{abstract}


and efficient numerical technique. The solution is compared to a finite difference and

lattice gas simulation and found to give more accurate results.

\section{Introduction}

A single-screw extruder is commonly used in polymer processing. The mixing performance of the extruder considerably influences the quality and morphology of the final product and so the flow field in the mixing section has been studies by a number of authors to gain a better understanding of the process. Yao at al. $(1996,1997)$ performed simulations using the finite difference method (FDM) which were found to compare favourably to a visualization experiment involving the flow of high viscosity corn syrup in a rectangular cavity with a moving top boundary. Further work was performed by Horiguchi et al. (2003) using the lattice gas method (LGM). Qualitatively the FDM and LGM determined the same oval-shaped flow pattern as was observed in the visualisation experiment. The simulations differed in that a small circulation was observed in the bottom left and right corners of the LGM simulations; whereas no circulation is evident at the bottom left hand corner in the FDM results. Horiguchi et al. (2003) also considered a quantitative comparison with theory. This indicated that the LGM produced a more accurate representation of the flow field compared to the FDM, however there was still a discrepancy between the LGM simulation and the theoretical expression for the velocity.

The success of the LGM in simulating flow in a single screw extruder has motivated the present study using the lattice Boltzmann model (LBM) which has been developed from the LGM in order to overcome a number of difficulties. The main aim of this study is to demonstrate the ability of the LBM to accurately simulate flow in the mixing section of a screw extruder. Using a coordinate system attached to the rotating screw, a screw extractor can be 
modeled as a cavity flow problem with one moving wall and three stationary walls. The velocity field can then be divided into two components: the two-dimensional cross-section component and the stream-wise component. One of the main applications of computer simulations is to investigate different geometries in order to maximise mixing. In this study a mixing section without pins was selected and the two-dimensional cross-section component considered to enable the result to be compared with alternative numerical, experimental and analytic results. (Yau et al., 1997; Horiguchi et al., 2003; McKelvey, 1962).

\section{Numerical Model}

The LBM (Chen and Doolen 1998) has evolved from the LGM. Despite the success of the LGM (Biggs and Humby, 1998; Rivet and Boon,2001), there are a number of difficulties in applying the LGM and this has lead to the development of the LBM. Below a number of these problems and their solution in the LBM are briefly considered, with particular attention to the simulations presented here and the work of Horiguchi et al. (2003) (HTY).

In the LGM individual 'particles' are considered to move on the underlying lattice. This leads to noisy results since at each grid point there can only be an integer number of particles and correspondingly only a discrete number of velocities are possible. This is generally overcome by averaging; HTY average over $32 \times 32$ square sites and over 500 time-steps, but there is still some evidence of noise in their plotted results. In the LBM a real valued distribution function is considered which was originally developed as an ensemble average of the LGM particle. This totally eliminates the noise and so either the grid size can be reduces significantly, or a greatly increased resolution is obtained. It is also not advisable to time-average if a problem without a steady-state solution is being simulated. 
In the LGM the kinematic viscosity, $\nu$, is a function of the fluid density. This can be problematic if the density is not constant. In general, even when the density remains constant throughout the simulation giving a constant value for the kinematic viscosity, the range of the kinematic viscosity is limited which in turn limits the range of the Reynolds number

$$
R e=\frac{U L}{\nu(\rho)}
$$

which can be achieved since the characteristic length $L$ is limited by the grid size which is in turn limited by the computational resources available; and the characteristic velocity scale $U$ is limited by the low Mach number condition $U \ll c$ (Frisch et al., 1987), where $c$ is the speed of sound (0.5 in HTY).

The equations of motion satisfied by the LGM are not the standard Navier-Stokes and continuity equations, but rather similar ones containing a density dependent function $g(\rho)$ (Frisch et al., 1987). In single phase flows this can be scaled out of the equations giving an altered Reynolds number (Wolfram 1986; Frisch et al., 1987)

$$
R e=\frac{g(\rho) U L}{\nu(\rho)}
$$

This additional factor is due to a lack of Galilean invariance in the LGM and is not present in the LBM. 


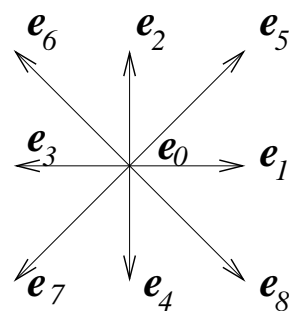

Figure 1: The lattice vectors at a grid site

\section{The lattice Boltzmann Model}

The LBM is well described in the literature, see for example (Chen and Doolen, 1998; Succi, 2001; Wolf-Gladrow, 2000). Here we give a brief description of the technique. The simulations are performed on a fixed underlying lattice, in the simulations presented here a square lattice with diagonals is used. Vectors $\boldsymbol{e}_{i}$, are defined along the eight link directions of the lattice for $i=1,2, \ldots ., 8$ as well as a null vector, $\boldsymbol{e}_{0}$, see figure 1 . At each site 9 distribution function are defined, one in each of the eight link directions of the lattice and a 'rest particle' distribution function. The distribution functions evolve with time and describe the fluid density, $\rho$, and velocity, $\boldsymbol{u}$, at each site:

$$
\rho(\boldsymbol{x}, t)=\sum_{i=0}^{i=8} f_{i}(\boldsymbol{x}, t) \quad \text { and } \quad \rho(\boldsymbol{x}, t) \boldsymbol{u}(\boldsymbol{x}, t)=\sum_{i=0}^{i=8} f_{i}(\boldsymbol{x}, t) \boldsymbol{e}_{i}
$$

Thus the fluid velocity can be found at each grid point and at each time-step from the distribution functions. In an LBM simulation the distribution functions evolve according to the Boltzmann equation (Chen and Doolen, 1998)

$$
f_{i}\left(\boldsymbol{r}+\boldsymbol{e}_{i}, t+1\right)-f_{i}(\boldsymbol{r}, t)=\frac{-1}{\tau}\left(f_{i}-\bar{f}_{i}\right)
$$

where

$$
\bar{f}_{i}(\boldsymbol{r}, t)=\rho\left(w_{i}+3 w_{i} \boldsymbol{e}_{i} \cdot \boldsymbol{u}+\frac{9}{2} w_{i}\left(\boldsymbol{e}_{i} \cdot \boldsymbol{u}\right)^{2}-\frac{3}{2} w_{i} u^{2}\right)
$$


and $w_{0}=4 / 9, w_{1}=w_{2}=w_{3}=w_{4}=1 / 9$ and $w_{5}=w_{6}=w_{7}=w_{8}=1 / 36$. The relaxation time, $\tau$, determines the kinematic viscosity of the fluid as

$$
\nu=\frac{2 \tau-1}{6} .
$$

Boundary conditions are also required. At moving walls the boundary conditions were applied following Zou and He (1997). At stationary walls half-way bounce back boundary conditions were applied, see for example Zou and He (1997); these give a boundary half way along the lattice link.

\section{Results and Discussion}

Simulations of a single screw extractor were performed using the LBM. Although the flow field in a single screw extruder consists of two components, only the flow field perpendicular to the spiral direction is considered. This is to enable a direct comparison with previous work and to enable comparison with theory. The computational grid had $L$ grid point in the $x$-direction and $H$ grid points in the $y$ direction and two difference sets of values for $L$ and $H$ were selected. One had $L=1536$ and $H=512$, the same size as HTK, but giving significantly greater resolution due to the averaging required with the LGM. The second simulation was performed with $L=49$ and $H=16$, giving the same resolution as HTK. The same viscosity was used in both sets of simulations with $\tau=0.83333$. The value of the wall velocity was then selected to achieve $R e=0.144$, giving $U=0.01$ and $U=0.00003125$ for the small and the large grids respectively. The flow was generated by the motion of the top wall $(y=H)$ with velocity $(\mathrm{U}, 0) ;$ no-slip boundary conditions were applied at the other three walls. 
To enable comparison between the LBM results and a real configuration, as with the LGM, it is necessary to compare dimensionless quantities: the Reynolds number $(U H / \nu)$, the dimensionless lengths $y^{*}=y / H$ and $x^{*}=x / H$ and the dimensionless velocity $\boldsymbol{u}^{*}=\boldsymbol{u} / U$, where $\boldsymbol{u}$ is the simulated velocity.

Figure 2 shows the flow field in the mixing section. Figure 2 (a) shows the magnitude of the velocity, $\left|u^{*}\right|=\sqrt{\left(u^{*}\right)^{2}+\left(v^{*}\right)^{2}}$. Also shown in figure 2 (a) are streamlines calculated from the velocity field. These are exactly symmetric about the centre of the $x$-axis as would be expected at this low Reynold's number. The velocity field is shown in figure 2 (b). Both figures show the same general features as Yao et al.(1996, 1997) and Horiguchi et al. (2003), however the results here do not show a small circulation at the bottom corners. Figure 3 shows the $x$-component of the the velocity, $u^{*}$ as a function of the $y$-position along a cross-section through the centre of the screw extractor. As well as the LBM results, computed on the smaller grid, the LGM and FDM results (Horiguchi et al. 2003), and the analytic solution (McKelvey, 1962):

$$
u^{*}=y^{*}\left(2-3 y^{*}\right)
$$

are also shown. The LBM results show very close agreement to the analytic solution, despite the small grid size. The results show a significant improvement over both the FDM and the LGM results. Compared to the LGM simulations, the use of a smaller grid increases the computational efficiency, both through the reduction in the number of grid points and through a steady state being achieved in less time-steps (around 100 compared to 100000 for the LGM). One of the main reasons for the improved accuracy of the LBM compared to the LGM is likely to be the Mach number of the simulations. In the LGM, despite the low 
Reynolds number, the lid velocity was 0.144 , giving a Mach number of 0.288 . In the LBM simulations on the smaller grid the Mach number was $1.73 \times 10^{-3}$. In both models the Mach number is required to be small if the system is to satisfy the Navier-Stokes equation (Frisch et al., 1987; Chen and Doolen, 1998). Errors due to compressibility scale as the square of the Mach number (Reider and Sterling, 1995; Martinez et al., 1994). It is also interesting to consider the differences between the FDM and LBM simulations. As identified by Horiguchi et al. (2003), there is a difference in the manner in which the boundary conditions are imposed. In the FDM method, fictitious boundary cells are defined and set at appropriate velocities. In the LBM the boundary conditions were implemented on the moving wall by calculating the unknown distribution functions following Zou and He (1997). This ensures the correct wall velocity is implemented and maintains the second-order accuracy of the simulation. The boundary conditions on the stationary walls were implemented using the same bounce-back rule as the LGM simulations (Horiguchi et al. 2003), but with the wall position half-way between the nodes. This ensures that, for the geometry used here, the stationary walls are also implemented with second-order accuracy. It is possible that these different approaches to the boundary conditions may explain the inconsistent results. Simulations of flow, such as those presented here, do not give quantitative measurements of mixing efficiency. Rather, the mixing efficiency is estimated by considering quantities derived from the velocity profile (Ottino and Ranz, 1981; Ottino 1989; Yao et al., 1997). An alternative approach which could be employed in an LBM simulation would be to simulate, for example, a binary fluid mixture and directly measure the mixing. Given the level of accuracy demonstrated here for a single phase LBM simulation, and the successful application of binary fluid LBM models in other fields (Rakotomalala et al., 1997; Buick and Greated, 1998; Pagonabarraga et al., 2001) such an approach warrants further investigation. 


\section{Conclusion}

The flow field in the mixing section of a single-screw extractor has been simulated using the LBM. The $x$-component of the velocity over a cross-section was compared with theory and with LGM and FDM simulations. The results show that the LBM provides a more accurate realisation of the flow field compared to previous simulations using the LGM and the FDM. The results also show no evidence of a small circulation in the bottom corners of the mixing section. The LBM has the same ability as the LGM to simulate flows in complex geometries, and so given its greater accuracy and flexibility we conclude that the LBM can be used as an effective numerical technique for analysing flow fields in industrial mixing.

\section{References}

[1] Biggs, M. J., S. J. Humby; "Lattice-Gas Automata Methods for Engineering", Trans. IChemE, 76A, 162-174 (1998).

[2] Buick, J. M. and C. A. Greated; "Lattice Boltzmann Modeling of Interfacial Waves", Phys. Fluids, 10, 1490-1511 (1998).

[3] Chen, S. and G. D. Doolen; "Lattice Boltzmann method for fluid flows", Annual Review of Fluid Mechanics, 30, 329-364 (1998).

[4] Frisch, U., D. d'Humières, B. Hasslacher , P. Lallemand, Y. Pomeau and J.-P. Rivet; "Lattice Gas Hydrodynamics in Two and Three Dimensions", Complex Systems, 1, 
[5] Horiguchi, H., K. Takahashi and T. Tokota; "Numerical Simulation of the Flow Field in the Mixing Section of a Screw Extruder by the Lattice Gas Automata Method", $J$. Chem. Eng. Japan, 36, 110-113 (2003).

[6] McKelvey, J. M.; Polymer Processing, pp. 228-247, Wiley, New York, USA (1962).

[7] , Martinez, D. O., W. H. Matthaeus, S. Chen and D. C. Montgomery, "Comparison of Spectral Method and Lattice Boltzmann Simulations of Two- Dimensional Hydrodynamics", Phys. Fluids, 6, 1285-1298 (1994).

[8] Ottino, J. M. and W. E. Ranz; "A framework for description of mechanical mixing of fluids", A.I.Ch.E. J., 27, 565-577 (1981).

[9] Ottino, J. M.; "The Kinematics of Maxing: Stretching, Chaos and Transport",64-95 Cambridge University Press, Cambridge, 1989.

[10] Pagonabarraga,I., J.-C. Desplat, A. J. Wagner and M. E. Cates; "Interfacial Dynamics in 3D Binary Fluid Demixing: Animation Studies", New J. Phys., 3, 9.1-9.18 (2001). 
[11] Rakotomatata, N., D. Salin and P. Watzky; "Miscible Displacement Between Two Parallel Plates: BGK Lattice Gas Simulations", J. Fluid Mech., 338, 277-297 (1997).

[12] Reider, M. B. and J. D. Sterling, "Accuracy of Discrete-Velocity BGK Models for the Simulation of the Incompressible Navier-Stokes Equations", Computers \& Fluids, 24, 459-467 (1995).

[13] Rivet, J.-P., J. P. Boon; Lattice Gas Hydrodunamics, Cambridge University Press (2001).

[14] Succi, S.; The Lattice Boltzmann Equation - For Fluid Dynamics and Beyond, Oxford University Press (2001).

[15] D. A. Wolf-Gladrow, D. A.; "Lattice-Gas Cellular Automata and Lattice Boltzmann Models", in Lecture Notes in Mathematics 1725, ed. Dold, A., F. Takens, F. and Teissier, B., Springer, 2000.

[16] Wolfram, S.; "Cellular Automaton Fluids 1: Basic Theory", Journal of Statistical Physics, 45, 471-529 (1986).

[17] Yao, W. G., K. Takahashi and Y. Abe; "Analytical Study on Flow and Distributive Mixing of a New Type Pin Mixing Section for Screw Extruder", Int. Poly. Proc., XI, 222-227 (1996). 
[18] Yao, W. G., K. Takahashi, K. Koyama and G. C. Dai; "Design of a New Type Pin Mixing Section for a Screw Extruder Based on Analysis of Flow and Distributive Mixing Performance", Chem. Eng. Sci., 52, 13-21 (1997).

[19] Zou, Q., X. He; "On pressure and velocity boundary conditions for the lattice Boltzmann BGK model"; Phys. Rev. E, 9; 1591-1598 (1997). 

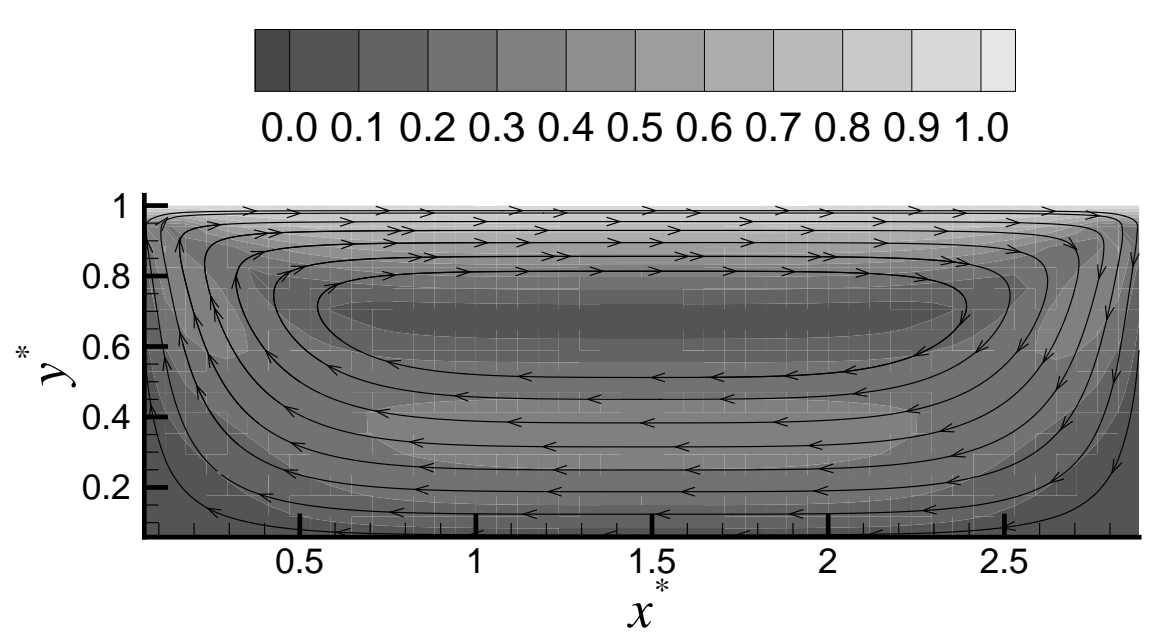

(a)

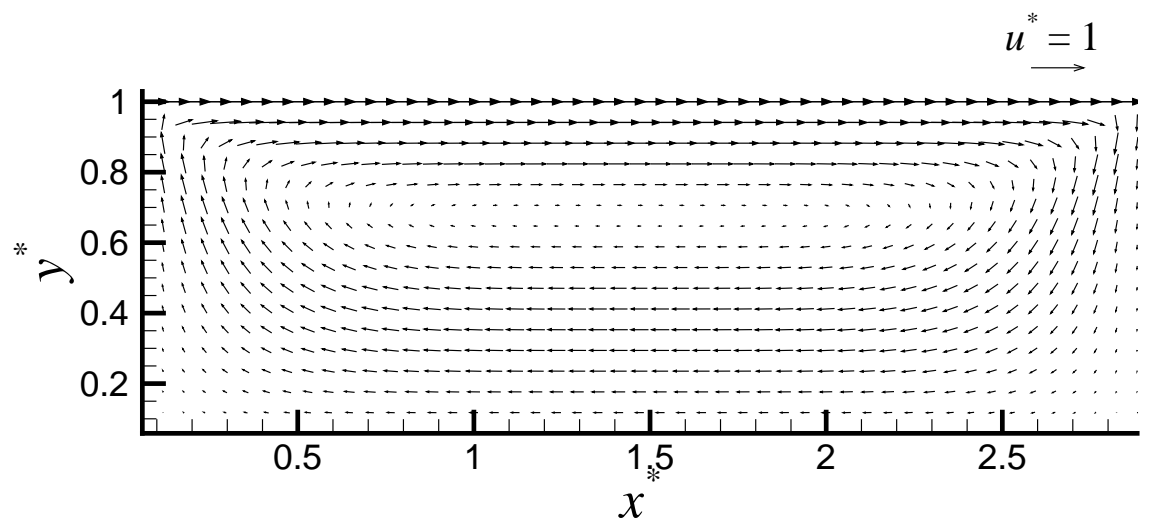

(b)

Figure 2: Flow pattern in the mixing section. (a) shows the magnitude of the dimensionless velocity with streamlines calculated from the flow and (b) shows the vector field. 


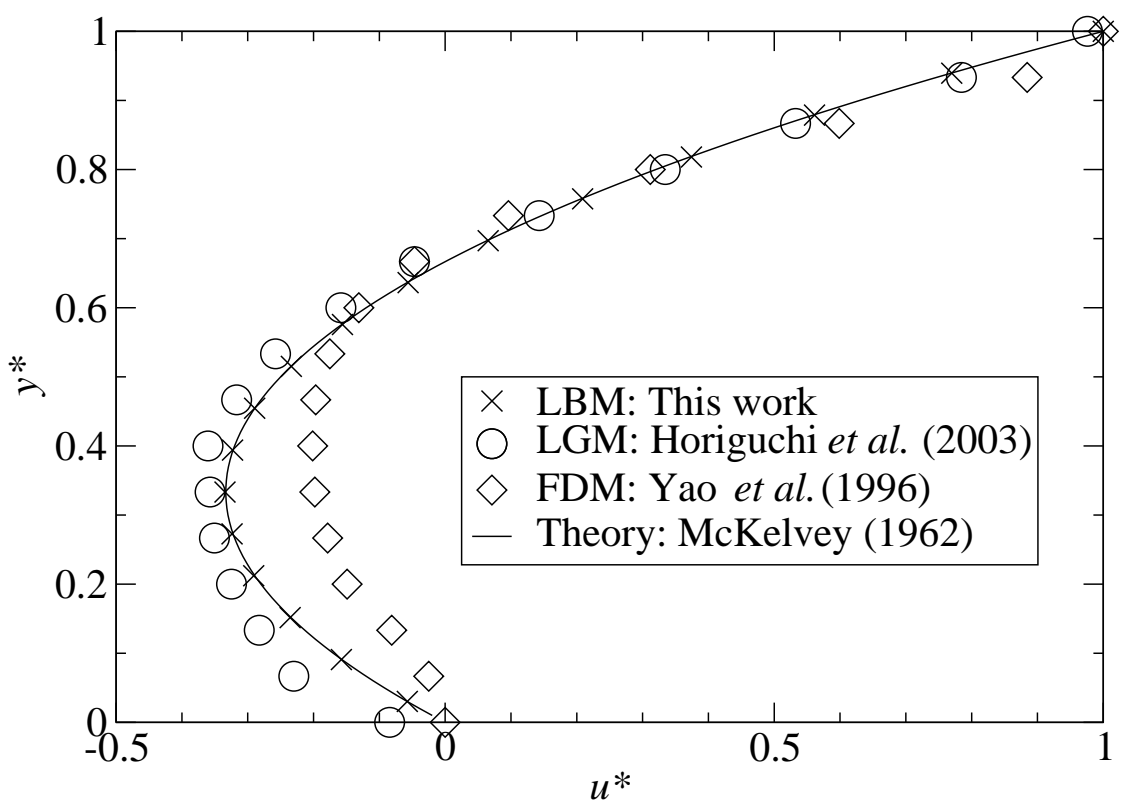

Figure 3: The normalised $x$-component of the velocity as a function of the normalised $y$-position along a section through the centre of the computational domain. 